\title{
One-step approach for solving general multi-objective De Novo programming problem involving fuzzy parameters
}

\author{
Susanta Banik (D), Debasish Bhattacharya* (D) \\ Mathematics Department, National Institute of Technology, Agartala, 799046, India
}

\begin{abstract}
Multi-objective De Novo Programming is a user-friendly device for optimal system design. There exist no method for solving general multi-objective De Novo Programs. Only some special cases have been discussed. This paper proposes a one-step method for solving a general De Novo Programming Problem using a Min-max Goal Programming technique where the parameters involved are all fuzzy numbers. The solution obtained is an efficient solution of the problem considered. The present approach is much more realistic than the standard De Novo Programming with crisp parameters. Two numerical examples are given to illustrate the solution procedure.
\end{abstract}

Mathematics Subject Classification (2010). 90C90, 90C70

Keywords. De Novo programming, fuzzy numbers, min-max goal programming, multi-objective programming, degree of possibility

\section{Introduction}

Traditional linear programming is a good way of obtaining the optimal allocation of fixed or limited resources. But the modern requirement has been shifted from an allocation of the fixed resources optimally in a given system to that of designing an optimal system extending the existed resources if necessary [25-30]. Zeleny [26] introduced the concept of De-Novo Programming to deal with optimal designing of a system where all the objectives are optimized simultaneously, no trade-offs among the objectives are necessary and no resources are left unused (i.e. all the constraints are satisfied in equality). This method was first designed for single-criterion decision making [26,28], later it has been extended to multi-criteria decision-making, containing the maximizing type of objectives only [27, $29,30]$. In the designed approach the computation could be handled easily and thus it has become a popular multi-criteria decision-making technique for designing an optimal system. But no method exists for the solution of general De Novo Programming Problems involving both maximizing and minimizing type of objectives. Only some special cases could be seen in the literature. To solve the general De Novo Programming Problems, Li and Lee [14,15], Chen and Hsieh [7], Nurullah [22], Chakraborty and Bhattacharya $[3,4]$ introduced several approaches. Li and Lee $[14,15]$ first introduced a two-phase fuzzy

\footnotetext{
*Corresponding Author.

Email addresses: susanta.banik580@gmail.com (S. Banik), bhattacharyad_nita2007@yahoo.co.in (D. Bhattacharya)

Received: 07.06.2018; Accepted: 25.08.2018
} 
approach based on the ideal and negative ideal solutions to solve the general De Novo Programming Problem. Chen and Hsieh [7] introduced an innovative approach to the solution of multi-stage general De Novo Programming Problems using a fuzzy dynamic programming concept. Nurullah [22] employed a Min-max Goal Programming technique for the solution of the multi-objective general De Novo Programming Problems in two steps and examined the closeness of the optimal objective values to the ideal values of the objectives. Chakraborty and Bhattacharya in $[3,4]$ further studied De Novo Programming Problem and proposed a method for the solution of the general De Novo Programming Problem in one step under fuzzy environment using Zimmermann's technique [32] with max-min operator. Shi [21] introduced several optimum-path ratios for enforcing different budget levels of resources so as to find alternative optimal system designs for solving multiobjective De Novo Programming (MDNP) Problems. Shi [20] applied MDNP to formulate and solve problems of system design that involved multiple decision makers and a possible debt. Also Babic and Pavic [1], Huang, et al. [9], Zhang et al. [31], and Chen and Tzeng [6] have contributed to the De Novo Programming literature with their studies. Miao et. al. [17] considered Interval-Fuzzy De Novo Programming method for planning water resources systems under uncertainty in 2014. In 2015 Saeedi et al. [19] utilized De Novo Programming technique to determine the capacity in a closed loop supply chain network when a queueing system is established at each recovery center in the reverse flow. Thus it can be said that De Novo Programming and its applications have become a vibrant area of contemporary research.

So far we have discussed De Novo Programs with all parameters (the coefficients of the variables) being crisp . Now in real world problems, determination of the coefficient of the variables appearing in the De Novo Program or other linear/ non-linear program with precision is rarely possible. So in absence of precise data, the decision maker has to be satisfied with available inaccurate data and this adversely affects the solution obtained. Due to this inherent uncertainty, Fuzzy De Novo Programming was introduced by Li and Lee $[14,15]$. In their construction all the parameters were taken as fuzzy numbers and a two-step approach for its solution was proposed. In this paper, we have proposed a one-step approach for solving general MDNP problem using min-max Goal Programming. In the proposed set up the parameters of costs, technological coefficients, unit prices of the resources and total budget are all taken as fuzzy numbers. The possibility theory introduced by Zadeh [24] has been proposed to transform the Fuzzy De Novo Programming Problem to an equivalent crisp problem using membership functions of the fuzzy parameters and the degree of possibility $\alpha \in[0,1]$ to which the solution satisfies the problem. After generating a crisp problem, a one-step technique using Min-max Goal Programming has been used to solve the problem for a given $\alpha$. Varying $\alpha$ one can plot the optimal objective values and can choose the most suitable one depending on the situation. To fulfil our aim the paper is organized as follows.

In Section 2, the basic concepts and formulations of the multi-objective De Novo Programming have been sketched. In Section 3, the Fuzzy De Novo Programming introduced by $\mathrm{Li}$ and Lee and its conversion to an equivalent crisp problem has been discussed. In Section 4, a Min-max Goal Programming approach has been applied to solve the crisp problem generated in Section 3. With aid of a theorem it has been established that the proposed solution method elicits an efficient solution of the problem in one-step. Two numerical examples are given to illustrate the approach. Finally, we address the conclusion in Section 5.

\section{De Novo programming problem}

In this section, we will discuss the basic concept of the De Novo Programming Problem suggested by Zeleny [26]. The general type of De Novo Programming Problem can be 
represented as

$$
\begin{aligned}
& \operatorname{Max} \mathrm{Z}=C^{1} \mathbf{x} \\
& \operatorname{Min} \mathrm{W}=C^{2} \mathbf{x}
\end{aligned}
$$

Subject to

$$
\begin{aligned}
& \mathbf{A x} \leq \mathbf{b} \\
& \sum_{i=1}^{m} p_{i} b_{i} \leq B \quad \mathbf{x} \geq 0
\end{aligned}
$$

where $C^{1}=\left[c_{k j}\right]_{r \times n} ; C^{2}=\left[c_{(l+r) j}\right]_{s \times n} ; \mathbf{A}=\left[a_{i j}\right]_{m \times n} ; \mathbf{b}^{T}=\left[b_{1}, b_{2}, \ldots, b_{m}\right]$;

$\mathbf{x}^{T}=\left[x_{1}, x_{2}, \ldots, x_{n}\right] ; \mathrm{Z}^{T}=\left[z_{1}, z_{2}, \ldots, z_{r}\right] ;$ and $\mathrm{W}^{T}=\left[w_{1}, w_{2}, \ldots, w_{s}\right]$, square brackets are used to denote row vectors.

Here, $\mathbf{x}, \mathbf{b}$, are the column vectors of decision variables for products and resources respectively, $p_{i}$ is the cost per unit of $i^{t h}$ resources, which are known, $i=1,2,3, \ldots, m$ and $B$ is the total available budget. Let $v_{j}=\sum_{i=1}^{m} p_{i} a_{i j}$ denote the unit cost of producing the product $j$. Then the De Novo Program (2.1) can be re-written as,

$$
\begin{aligned}
& \operatorname{Max} \mathrm{Z}=C^{1} \mathbf{x} \\
& \operatorname{Min} \mathrm{W}=C^{2} \mathbf{x}
\end{aligned}
$$

Subject to

$$
\begin{aligned}
& \sum_{j=1}^{n} v_{j} x_{j} \leq B \\
& x_{j} \geq 0, \quad j=1,2, . ., n
\end{aligned}
$$

The problem has been reduced to an equivalent problem containing only one constraint involving $\mathrm{n}$ arguments. It can be proved that every solution of the system (2.1) is also a solution of the system (2.2) and conversely [26]. Thus with these restructuring, the generality of the system is not disturbed. In this paper, we are concerned with fuzziness in the above system and design a problem in which all the parameters, $c_{k j}, c_{(l+r) j}, p_{i}, a_{i j}, B$ are fuzzy numbers.

\section{Fuzzy De Novo programming}

Like traditional linear or non-linear multi-objective programs, standard De Novo Programming also requires specifically known data structures. But in the real world, it is often difficult to determine the coefficient of the variables accurately. Li and Lee [14,15] introduced a fuzzy environment into the general model of the De Novo programming to make standard De Novo Programming more flexible as follows: 


$$
\begin{array}{lll}
\operatorname{Max} & \tilde{Z}_{k}=\sum_{j=1}^{n} \tilde{C}_{k j} x_{j} & k=1,2, \ldots, r . \\
\operatorname{Min} & \tilde{W}_{l}=\sum_{j=1}^{n} \tilde{C}_{(l+r) j} x_{j} & l=1,2, \ldots, s .
\end{array}
$$

Subject to

$$
\begin{aligned}
& \sum_{j=1}^{n} \tilde{v}_{j} x_{j} \leq \tilde{B} \\
& \tilde{v}_{j}=\sum_{i=1}^{m} \tilde{p}_{i} \tilde{a}_{i j} \\
& x_{j} \geq 0, \quad j=1,2, \ldots, n
\end{aligned}
$$

where parameters $\tilde{C}_{k j}, \tilde{C}_{(l+r) j}, \tilde{p}_{i}, \tilde{a}_{i j}, \tilde{B}$ are fuzzy numbers on the set of real numbers $\mathcal{R}$ characterized by the membership functions $\mu_{\tilde{C}_{k j}}, \mu_{\tilde{C}_{(l+r) j}}, \mu_{\tilde{p}_{i}}, \mu_{\tilde{a}_{i j}}, \mu_{\tilde{B}}$ respectively, and $\tilde{v}_{j}$ are fuzzy functions on $\mathcal{R}^{2 m}$ defined by using an extension principle,

$$
\mu_{\tilde{v}_{j}}\left(v_{j}\right)=\sup _{\sum_{i=1}^{m} p_{i} a_{i j}=v_{j}} \min _{i}\left\{\mu_{\tilde{p}_{i}}\left(p_{i}\right), \mu_{\tilde{a}_{i j}}\left(a_{i j}\right)\right\} \quad j=1,2, \ldots, n .
$$

In (3.1) above $\tilde{Z}_{k}$ and $\tilde{W}_{l}$ are fuzzyfied versions of $Z_{k}$ and $W_{l}$ respectively. By means of an extension principle we have,

$$
\begin{gathered}
\mu_{\tilde{Z}_{k}}(\mathbf{x})=\sup _{Z_{k}=\sum_{j=1}^{n} C_{k j} x_{j}} \min _{j}\left\{\mu_{\tilde{C}_{k j}}\left(C_{k j}\right)\right\} ; k=1,2, \ldots, r ; \mathbf{x}=\left(x_{1}, x_{2}, \ldots, x_{n}\right) \\
\mu_{\tilde{W}_{l}}(\mathbf{x})=\sup _{W_{l}=\sum_{j=1}^{n} C_{(l+r) j} x_{j}} \min _{j}\left\{\mu_{\tilde{C}_{(l+r) j}}\left(C_{(l+r) j}\right)\right\} ; l=1,2, \ldots, s \\
\mu_{\left(\sum_{j=1}^{n} \tilde{v}_{j} x_{j} \leq \tilde{B}\right)}(\mathbf{x})=\sup _{\sum_{j=1}^{n} \tilde{v}_{j} x_{j} \leq B} \min _{j}\left\{\mu_{\tilde{v}_{j}}\left(v_{j}\right), \mu_{\tilde{B}}(B)\right\} \\
=\operatorname{lip}_{j=1}^{n} \tilde{v}_{j} x_{j} \leq B \\
\min _{j}\left\{\sup _{v_{j}=\sum_{i=1}^{m} p_{i} a_{i j}} \min \left\{\mu_{\tilde{p}_{i}}\left(p_{i}\right), \mu_{\tilde{a}_{i j}}\left(a_{i j}\right)\right\}, \mu_{\tilde{B}}(B)\right\}
\end{gathered}
$$

Here $\mu_{\tilde{Z}_{k}}, \mu_{\tilde{W}_{l}}, \mu_{\left.\sum_{j=1}^{n} \tilde{v}_{j} x_{j} \leq \tilde{B}\right)}$ are fuzzy sets on $X$, the set of all feasible solutions of (3.1) and $\mathbf{x} \in X$. Each of these fuzzy sets serves as fuzzy restriction on the variable $\mathbf{x}=$ $\left(x_{1}, x_{2}, \ldots x_{n}\right)$, in the sense that the assignment of the values $\mathbf{u}=\left(u_{1}, u_{2}, \ldots, u_{n}\right)$ to $\mathbf{x}$ has the form

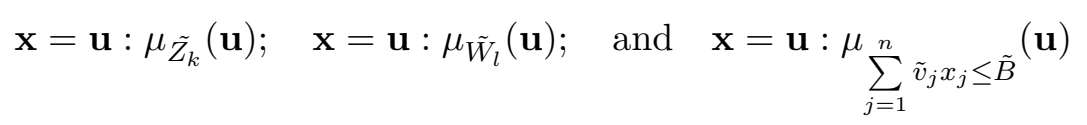

where, $\mu_{\tilde{Z}_{k}}$ etc. are the degree to which the constraint represented by $\tilde{Z}_{k}$ is satisfied when $\mathbf{u}$ is assigned to $\mathbf{x}$. Equivalently it implies that $1-\mu_{\tilde{Z_{k}}}(\mathbf{u})$ etc. are the degrees to which the constraint has to be stretched in order to allow the assignment of the values $\mathbf{u}$ to $\mathbf{x}$. In other words, $\mu_{\tilde{Z_{k}}}$ etc. are the degrees of possibility to which the fuzzy restriction $\tilde{Z}_{k}$ is 
satisfied. Then we take

$$
\alpha=\min \left\{\operatorname{poss}\left(\tilde{Z}_{k}\right), \operatorname{poss}\left(\tilde{W}_{l}\right), \operatorname{poss}\left(\sum_{j=1}^{n} \tilde{v}_{j} x_{j} \leq \tilde{B}\right)\right\}
$$

where, $k=1,2, \ldots, r ; \quad l=1,2, \ldots, s$. Here poss denotes possibility and $\alpha \in[0,1]$ represents the degree of possibility to which the solution satisfies the problem (3.1). In other words, it can be said that $\alpha \in[0,1]$ is the safety level or efficiency level and $1-\alpha$ is the risk-factor [15]. Also $\operatorname{poss}\left(\tilde{Z}_{k}\right)=\mu_{\tilde{Z}_{k}}$ etc.

Now for designing a risk-free system under fuzzy environment, the parameters viz. invested budget $(\tilde{B})$, unit resource price $\left(\tilde{p}_{i}\right)$, and operation cost $\left(\tilde{a}_{i j}\right)$ are respectively taken as following types of interval $\left[B^{0}, B^{1}\right),\left(p^{1}, p^{0}\right],\left(a^{1}{ }_{i j}, a^{0}{ }_{i j}\right]$, where the superscript 0 corresponds to risk free (i.e. having membership value 1 at the corresponding end of the interval) and the superscript 1 to the impossible value (i.e. having membership value 0 at the corresponding end of the interval). The cost coefficient $\tilde{C}_{k j}$ of the maximizing type of objectives are taken as $\left[C^{0}{ }_{k j}, C^{1}{ }_{k j}\right)$ and for the minimizing type of objectives the cost coefficient $\tilde{C}_{(l+r) j}$ are taken as $\left(C^{1}{ }_{(l+r) j}, C^{0}{ }_{(l+r) j}\right]$. In all these cases, between the risk-free and impossible value we take a Zimmerman type of linear membership function. On the other hand, a system designed with possible biggest profit units for maximizing types of objectives (respectively possible smallest cost unit for the minimizing types of objectives), biggest invested budget, smallest resources price and smallest operation cost are most dangerous $[2,15]$.

Now as in $[14,15,23]$ using the definition of $\alpha$, the program (3.1) becomes,

$$
\begin{array}{rlrl}
\operatorname{Max}\left(\tilde{Z}_{k}\right)_{\alpha} & =\sum_{j=1}^{n} \mu_{\tilde{C}_{k j}}^{-1}(\alpha) x_{j} & k=1,2, \ldots, r \\
\operatorname{Max}\left(\tilde{W}_{l}\right)_{\alpha}=\sum_{j=1}^{n} \mu_{\tilde{C}_{(l+r) j}}^{-1}(\alpha) x_{j} & l=1,2, \ldots, s
\end{array}
$$

Subject to,

$$
\begin{aligned}
& \sum_{j=1}^{n} \mu_{\tilde{v}_{j}}^{-1}(\alpha) x_{j} \leq \mu_{\tilde{B}}^{-1}(\alpha) \\
& \alpha \in[0,1], \quad x_{j} \geq 0 ; \quad j=1,2, \ldots, n .
\end{aligned}
$$

where $\left(\tilde{Z}_{k}\right)_{\alpha},\left(\tilde{W}_{l}\right)_{\alpha}$, are the $\alpha$-cuts of $\tilde{Z}_{k}$ and $\tilde{W}_{l}$ defined by

$$
\begin{aligned}
& \left(\tilde{Z}_{k}\right)_{\alpha}=\left\{Z_{k} \in \mathcal{R} \mid \mu_{\tilde{Z}_{k}}\left(Z_{k}\right) \geq \alpha\right\}, \quad k=1,2, \ldots, r \\
& \left(\tilde{W}_{l}\right)_{\alpha}=\left\{W_{l} \in \mathcal{R} \mid \mu_{\tilde{W}_{l}}\left(W_{l}\right) \geq \alpha\right\}, \quad l=1,2, \ldots, s
\end{aligned}
$$

And

$$
\begin{array}{lr}
\mu_{\tilde{C}_{k j}}^{-1}(\alpha)=C^{1}{ }_{k j}-\alpha\left(C^{1}{ }_{k j}-C^{0}{ }_{k j}\right), & k=1,2, \ldots, r ; j=1,2, \ldots, n \\
\mu_{\tilde{C}_{(l+r) j}}^{-1}(\alpha)=C^{1}{ }_{(l+r) j}+\alpha\left(C^{0}{ }_{(l+r) j}-C^{1}{ }_{(l+r) j}\right), & l=1,2, \ldots, s ; j=1,2, \ldots, n \\
\mu_{\tilde{p}_{i}}^{-1}(\alpha)=p^{1}{ }_{i}+\alpha\left(p^{0}{ }_{i}-p^{1}{ }_{i}\right), & i=1,2, \ldots, m \\
\mu_{\tilde{a}_{i j}}^{-1}(\alpha)=a^{1}{ }_{i j}+\alpha\left(a^{0}{ }{ }_{i j}-a^{1}{ }_{i j}\right), & \\
\mu_{\tilde{B}}^{-1}(\alpha)=B^{1}-\alpha\left(B^{1}-B^{0}\right) & \\
\mu_{\tilde{v}_{j}}^{-1}(\alpha)=\sum_{i=1}^{m} \mu_{\tilde{p}_{i}}{ }^{1}(\alpha) \mu_{\tilde{a}_{i j}}^{-1}(\alpha) & j=1,2, \ldots, m ; j=1,2, \ldots, n
\end{array}
$$


Thus the fuzzy system (3.1) has been converted to an equivalent crisp system (3.2) (Verdegay [23]), for a given $\alpha \in[0,1]$. Our aim is to solve this crisp problem using Min-max Goal Programming technique, which requires calculation of target values/ aspiration levels of the objective. Here by aspiration levels of the objectives, we meant the targeted values of the objectives set by the decision makers for their achievement. In our calculations for a maximizing(resp. minimizing) type of objective, its maximum (resp. minimum) value under the given constraints has been taken as its aspiration level. For this, we first find the basic feasible solutions of the system. This is done by putting $(n-1)$ variables to zero and finding the value of the remaining variable.

Thus the basic solutions are,

$$
\left(\frac{B(\alpha)}{v_{1}(\alpha)}, 0, \ldots, 0\right) ;\left(0, \frac{B(\alpha)}{v_{2}(\alpha)}, 0, \ldots, 0\right) ; \ldots ;\left(0,0, \ldots, \frac{B(\alpha)}{v_{n}(\alpha)}\right) ;
$$

Here $B(\alpha)=\mu_{\tilde{B}}^{-1}(\alpha) \geq 0$ and $v_{j}(\alpha)=\mu_{\tilde{v}_{j}}^{-1}(\alpha)>0$. So all the basic solutions are feasible. Substituting these basic feasible solutions one by one in all the objectives we can find the optimum values of each of the objectives. Let

$$
\begin{array}{rlrl}
Z_{k}^{*} & =\operatorname{Max}\left(\tilde{Z}_{k}\right)_{\alpha}, & & k=1,2, \ldots, r \\
W_{l}^{*} & =\operatorname{Min}\left(\tilde{W}_{l}\right)_{\alpha}, & l & l=1,2, \ldots, s .
\end{array}
$$

These values $Z_{k}^{*}, W_{l}^{*}$ are called ideal values for the maximizing and minimizing objectives respectively and serve as the target values/aspiration levels for the objectives.

The $(l+r)$ component vector $I^{*}=\left(Z_{1}^{*}, Z_{2}^{*}, \ldots, Z_{r}^{*}, W_{1}^{*}, W_{2}^{*}, \ldots, W_{s}^{*}\right)$ of the ideal values of the objectives, is called the ideal point.

In Min-max goal programming, maximum possible deviations of the objectives from their respective ideal values are minimized. But the objective may have different units. To get rid of this difficulty, the objectives are made dimensionless through a normalization procedure. To apply the normalization procedure, another set of objective values are needed. For this purpose, Li and Lee [14] used negative ideal values given by $Z_{k}^{-}=\operatorname{Min}\left(Z_{k}\right)_{\alpha}$ and $W_{s}^{-}=\operatorname{Max}\left(W_{s}\right)_{\alpha}$ which denote the worst possible performance of the system.

In this paper, instead of using the negative ideal values, another set of objective values calculated by Luhandjula's comparison technique [16] have been used. These intermediate values (between the ideal and negative ideal value) of the objectives constitute the pessimistic objective values in the present case.

Let $\hat{\beta}=\left(\hat{Z}_{1}, \hat{Z}_{2}, \ldots, \hat{Z}_{r}, \hat{W}_{1}, \hat{W}_{2}, \ldots ., \hat{W}_{s}\right)$ be the vector of the pessimistic values.

\section{Min-max goal programming}

Goal Programming (GP) is one of the most important methods in the arena of Multi-Objective Decision Making (MODM). This method is regarded as an extension of classical linear programs which includes the achievement of target values or aspiration levels for each objective, in place of maximizing or minimizing the objective functions. The term Goal Programming was first introduced by Charnes and Cooper [5]. With the studies of Lee [13], Ijiri [11], and Ignizio [10]; Goal Programming has become a strong and well-accepted technique in the literature. The general purpose of Goal Programming is to minimize the deviation between the achievement of the goals and their aspirational levels. The minimization procedure can be accomplished with different methods (Romero [18]). The min-max variant of goal programming technique was introduced by Flavell [8]. In this variant, the maximum deviation from amongst the weighted set of deviations (of the objectives from their respective aspiration level) is minimized rather than the sum of the deviations themselves (Jones and Tamiz [12]). The mathematical expression of min-max GP is as follows: 
Min $d$

Subject to

$$
\begin{aligned}
& f_{k}(\mathbf{x})+n_{k}-p_{k}=w_{k} \\
& \beta_{k} n_{k}+\gamma_{k} p_{k} \leq d \\
& \mathbf{x} \in X, \quad \text { the set of all feasible solution of the problem } \\
& n_{k}, p_{k} \geq 0, \quad k=1,2, \ldots, r ; \quad \mathbf{x}^{\mathbf{T}}=\left[x_{1}, x_{2}, \ldots, x_{n}\right]
\end{aligned}
$$

where $d$ is the maximum weighted deviation between the achievement of the goals and their aspirational levels, $f_{k}(\mathbf{x})$ is the $k^{t h}$ objective function, $w_{k}$ is the specified aspiration level for the $k^{t h}$ objective $\left(\max / \min f_{k}\right), n_{k}, p_{k}$ are respectively negative and positive deviations from the aspiration level $w_{k}$ of $k^{\text {th }}$ objective and $\beta_{k}, \gamma_{k}$ are the non-negative weights with $\beta_{k}+\gamma_{k}=1$. For obtaining a satisfactory solution of the De Novo Programming Problem based on Min-max Goal Programming, the problem (3.2) is reformulated. According to the new formulation $w_{k}=$ ideal value of the $k^{\text {th }}$ maximizing / minimizing objective. Since the ideal values have been used as aspiration level of the objectives, so $f_{k}(\mathbf{x}) \leq Z_{k}^{*}$ for maximizing objectives and $f_{l}(\mathbf{x}) \geq W_{l}^{*}$, for minimizing objectives. Hence we must have $p_{k}=0$ for the $k^{t h}$ maximizing objective and $n_{l}=0$ for the $l^{\text {th }}$ minimizing objective. Now the ideal values (optimistic values $Z_{k}^{*}, W_{l}^{*}$ ) and pessimistic values $\left(\hat{Z}_{k}, \hat{W}_{k}\right)$ obtained by Luhandjula's comparison technique [16] will be used for the normalization of deviation variables. In normalization process each deviation variable is divided by a constant pertaining to the corresponding objective. This will reduce the deviations to unitless numbers. In this paper, this normalization constant has been taken as $\left(Z_{k}^{*}-\hat{Z}_{k}\right)$ [resp. $\left(\hat{W}_{l}-W_{l}^{*}\right)$ ] for maximizing [resp. for minimizing] objectives.

Now for solving the crisp De Novo Programming Problem (3.2) by using Min-max Goal Programming method (4.1) we have the following program.

$$
\begin{aligned}
& \text { Min } d \\
& \text { Subject to } \\
& \left(\tilde{Z}_{k}\right)_{\alpha}+n_{k}=Z_{k}^{*} \\
& \beta_{k} \frac{n_{k}}{t_{k}} \leq d \\
& \left(\tilde{W}_{l}\right)_{\alpha}-p_{l}=W_{l}^{*} \\
& \gamma_{l} \frac{p_{l}}{t_{l}} \leq d \\
& \sum_{j=1}^{n} \mu_{\tilde{v}_{j}}^{-1}(\alpha) x_{j} \leq \mu_{\tilde{B}}^{-1}(\alpha) \\
& \alpha \in[0,1], \quad x_{j} \geq 0 ; \quad j=1,2, \ldots, n .
\end{aligned}
$$

Now we will show with the help of a Theorem that the proposed method of solution (program 4.2) indeed yields an efficient solution of the problem (3.2). 
Theorem 4.1. The optimal solution of the system (4.2) is an efficient solution of the problem (3.2)

Assume $\overline{\mathbf{x}}$ be an optimal solution of system (4.2). We will show that $\overline{\mathbf{x}}$ is an efficient solution of system (3.2). If possible let, $\overline{\mathbf{x}}$ is not an efficient solution of (3.2).

Let $X=\left\{\mathbf{x} \in R^{n}, \mid \quad \sum_{j=1}^{n} \mu_{\tilde{v}_{j}}^{-1}(\alpha) x_{j} \leq \mu_{\tilde{B}}^{-1}(\alpha) ; \quad \alpha \in[0,1]\right\}$ Then $\exists \quad \overline{\overline{\mathbf{x}}} \in X$ such that,

$$
\begin{aligned}
& \left(\tilde{Z}_{k}\right)_{\alpha}(\overline{\overline{\mathbf{x}}}) \geq\left(\tilde{Z}_{k}\right)_{\alpha}(\overline{\mathbf{x}}) ; \quad \text { for } \quad k=1,2, \ldots, r \\
& \left(\tilde{W}_{l}\right)_{\alpha}(\overline{\overline{\mathbf{x}}}) \leq\left(\tilde{W}_{l}\right)_{\alpha}(\overline{\mathbf{x}}) ; \quad \text { for } \quad l=1,2, \ldots, s
\end{aligned}
$$

And for at least one $k$ or one $l$

$$
\left(\tilde{Z}_{k}\right)_{\alpha}(\overline{\overline{\mathbf{x}}})>\left(\tilde{Z}_{k}\right)_{\alpha}(\overline{\mathbf{x}}) ; \quad\left(\tilde{W}_{l}\right)_{\alpha}(\overline{\overline{\mathbf{x}}})<\left(\tilde{W}_{l}\right)_{\alpha}(\overline{\mathbf{x}}) ;
$$

Now let $F=\left\{\mathbf{x} \in X \mid \quad\left(\tilde{Z}_{k}\right)_{\alpha}(\mathbf{x})>\hat{Z}_{k} ;\left(\tilde{W}_{l}\right)_{\alpha}(\mathbf{x})<\hat{W}_{l} \quad \forall \quad k, s\right\}, F \neq \phi, \overline{\mathbf{x}} \in F$.

Here $\hat{Z}_{k}$ and $\hat{W}_{l}$ are respectively pessimistic value for $k^{\text {th }}$ maximizing objective and $l^{\text {th }}$ minimizing objective. Now we claim that $\overline{\overline{\mathbf{x}}}$ can not be in $X-F$. Otherwise, there will be at least one $k$ or one $l$ such that

$$
\left(\tilde{Z}_{k}\right)_{\alpha}(\overline{\overline{\mathbf{x}}}) \leq\left(\hat{Z}_{k}\right) \quad \text { or } \quad\left(\tilde{W}_{l}\right)_{\alpha}(\overline{\overline{\mathbf{x}}}) \geq\left(\hat{W}_{l}\right)
$$

Now since $\overline{\mathbf{x}}$ is an optimal solution of system (4.2), therefore for $\forall k$ and $l$,

$$
\left(\tilde{Z}_{k}\right)_{\alpha}(\overline{\mathbf{x}})>\left(\hat{Z}_{k}\right) \text { and }\left(\tilde{W}_{l}\right)_{\alpha}(\overline{\mathbf{x}})<\left(\hat{W}_{l}\right) .
$$

Now combining $\left(A_{1}\right)$ and $\left(B_{1}\right)$ we get,

$$
\left(\tilde{Z}_{k}\right)_{\alpha}(\overline{\overline{\mathbf{x}}})<\left(\tilde{Z}_{k}\right)_{\alpha}(\overline{\mathbf{x}}) \text { or } \quad\left(\tilde{W}_{l}\right)_{\alpha}(\overline{\overline{\mathbf{x}}})>\left(\tilde{W}_{l}\right)_{\alpha}(\overline{\mathbf{x}}),
$$

which is a conradiction. Thus $\overline{\overline{\mathbf{x}}} \in F$.

Now from the system (4.2) we can write the normalized values of $n_{k}$ and $p_{l}$,

$$
\frac{n_{k}}{t_{k}}=\frac{Z_{k}^{*}-\left(\tilde{Z}_{k}\right)_{\alpha}}{Z_{k}^{*}-\hat{Z}_{k}} ; \quad \frac{p_{l}}{t_{l}}=\frac{\left(\tilde{W}_{l}\right)_{\alpha}-W_{l}^{*}}{\hat{W}_{l}-W_{l}^{*}}
$$

Now,

Therefore,

$$
\left(\tilde{Z}_{k}\right)_{\alpha}(\overline{\overline{\mathbf{x}}}) \geq\left(\tilde{Z}_{k}\right)_{\alpha}(\overline{\mathbf{x}}) ; \quad k=1,2, \ldots, r .
$$

$$
\frac{Z_{k}^{*}-\left(\tilde{Z}_{k}\right)_{\alpha}(\overline{\overline{\mathbf{x}}})}{Z_{k}^{*}-\hat{Z}_{k}} \leq \frac{Z_{k}^{*}-\left(\tilde{Z}_{k}\right)_{\alpha}(\overline{\mathbf{x}})}{Z_{k}^{*}-\hat{Z}_{k}}
$$

Also,

$$
\left(\tilde{W}_{l}\right)_{\alpha}(\overline{\overline{\mathbf{x}}}) \leq\left(\tilde{W}_{l}\right)_{\alpha}(\overline{\mathbf{x}}) \quad l=1,2, \ldots, s .
$$

and

$$
\frac{\left(\tilde{W}_{l}\right)_{\alpha}(\overline{\overline{\mathbf{x}}})-W_{l}^{*}}{\hat{W}_{l}-W_{l}^{*}} \leq \frac{\left(\tilde{W}_{l}\right)_{\alpha}(\overline{\mathbf{x}})-W_{l}^{*}}{\hat{W}_{l}-W_{l}^{*}}
$$

and for at least one $k$, and one $l$,

$$
\frac{Z_{k}^{*}-\left(\tilde{Z}_{k}\right)_{\alpha}(\overline{\overline{\mathbf{x}}})}{Z_{k}^{*}-\hat{Z}_{k}}<\frac{Z_{k}^{*}-\left(\tilde{Z}_{k}\right)_{\alpha}(\overline{\mathbf{x}})}{Z_{k}^{*}-\hat{Z}_{k}} .
$$

And,

$$
\frac{\left(\tilde{W}_{l}\right)_{\alpha}(\overline{\overline{\mathbf{x}}})-W_{l}^{*}}{\hat{W}_{l}-W_{l}^{*}}<\frac{\left(\tilde{W}_{l}\right)_{\alpha}(\overline{\mathbf{x}})-W_{l}^{*}}{\hat{W}_{l}-W_{l}^{*}}
$$


Now for any arbitrary point $\mathbf{x} \in X, \min d=\min \left\{\beta_{k} \frac{n_{k}}{t_{k}}, \gamma_{l} \frac{p_{l}}{t_{l}}\right\}$. Since this is true for all $\beta_{k}$ and $\gamma_{l}$, we can choose $\beta_{k}=\gamma_{l}$. Now from $\left(A_{2}\right)$ and $\left(B_{2}\right)$ it is clear that the value of $\frac{n_{k}}{t_{k}}$ and $\frac{p_{l}}{t_{l}}$ is greater for $\overline{\mathbf{x}}$ than that at $\overline{\overline{\mathbf{x}}}$. Hence the minimum value of $d=\min \left\{\frac{n_{k}}{t_{k}}, \frac{p_{l}}{t_{l}}\right\}$ will occur at $\overline{\overline{\mathbf{x}}}$, which contradicts the fact that $\overline{\mathbf{x}}$ is an optimum solution of the system (4.2). Therefore $\overline{\mathbf{x}}$ is an efficient solution.

The advantage of this approach is that the decision maker is allowed to participate in the decision process by choosing his/her appropriate membership grade based on the risk factor, he/she is willing to take. The following example is taken from Li and Lee [14] (example 3, page 109) to illustrate the proposed approach.

\section{Example: 1}

Subject to

$$
\begin{array}{ll}
\operatorname{Max} & \tilde{Z}_{1}=\left[2^{0}, 5^{1}\right) x_{1}+12 x_{2} \\
\operatorname{Max} & \tilde{Z}_{2}=4 x_{1}+\left[1^{0}, 3^{1}\right) x_{2} \\
\operatorname{Min} & \tilde{W}_{1}=x_{1}+\left(1^{1}, 4^{0}\right] x_{2} \\
\operatorname{Min} & \tilde{W}_{2}=2 x_{1}+\left(2^{1}, 3^{0}\right] x_{2}
\end{array}
$$

$$
\begin{aligned}
& \left(\left(0.5^{1}, 2^{0}\right]+2\right) x_{1}+\left(\left(0.5^{1}, 2^{0}\right] \cdot\left(1^{1}, 4^{0}\right]+\left(2^{1}, 3^{0}\right]\right) x_{2} \leq\left[200^{0}, 250^{1}\right) . \\
& \quad x_{1}, x_{2} \geq 0
\end{aligned}
$$

For a given safety factor $\alpha$, the $\alpha$-cuts of the fuzzy coefficients can be expressed by means of inverses of their respective membership functions as presented in page 6; under system $(3.2)$

$$
\begin{array}{r}
\mu_{\tilde{C}_{11}}^{-1}(\alpha)=5-3 \alpha, \quad \mu_{\tilde{C}_{12}}^{-1}(\alpha)=12, \quad \mu_{\tilde{C}_{21}}^{-1}(\alpha)=4, \quad \mu_{\tilde{C}_{22}}^{-1}(\alpha)=3-2 \alpha, \\
\mu_{\tilde{C}_{31}}^{-1}(\alpha)=1, \quad \mu_{\tilde{C}_{32}}^{-1}(\alpha)=1+3 \alpha, \quad \mu_{\tilde{C}_{41}}^{-1}(\alpha)=2, \quad \mu_{\tilde{a}_{42}}^{-1}(\alpha)=2+\alpha, \\
\mu_{\tilde{a}_{11}}^{-1}(\alpha)=1, \quad \mu_{\tilde{a}_{12}}^{-1}(\alpha)=1+3 \alpha, \quad \mu_{\tilde{a}_{21}}^{-1}(\alpha)=2, \quad \mu_{\tilde{a}_{22}}^{-1}(\alpha)=2+\alpha, \\
\mu_{\tilde{p}_{1}}^{-1}(\alpha)=0.5+1.5 \alpha, \quad \mu_{\tilde{p}_{2}}^{-1}(\alpha)=1, \quad \mu_{\tilde{B}}^{-1}(\alpha)=250-50 \alpha, \\
\mu_{\tilde{v}_{1}}^{-1}(\alpha)=2.5+1.5 \alpha, \quad \mu_{\tilde{v}_{2}}^{-1}(\alpha)=2.5+4 \alpha+4.5 \alpha^{2} .
\end{array}
$$

Now the problem (4.3) can be re-written as a crisp program

$$
\begin{array}{ll}
\operatorname{Max} & \left(\tilde{Z}_{1}\right)_{\alpha}=(5-3 \alpha) x_{1}+12 x_{2} \\
\operatorname{Max} & \left(\tilde{Z}_{2}\right)_{\alpha}=4 x_{1}+(3-2 \alpha) x_{2} \\
\operatorname{Min} & \left(\tilde{w}_{1}\right)_{\alpha}=x_{1}+(1+3 \alpha) x_{2} \\
\operatorname{Min} & \left(\tilde{W}_{2}\right)_{\alpha}=2 x_{1}+(2+\alpha) x_{2}
\end{array}
$$

Subject to

$$
\begin{aligned}
& (2.5+1.5 \alpha) x_{1}+\left(2.5+4 \alpha+4.5 \alpha^{2}\right) x_{2}=250-50 \alpha \\
& \alpha \in[0,1], \quad x_{1}, x_{2} \geq 0
\end{aligned}
$$

For a given safety level $\alpha \in[0,1]$ one can solve the problem (4.4) using the Min-max program (4.2) proposed in this paper. Considering the value of $\alpha$ as 
$0.1,0.2,0.3,0.4,0.5,0.6,0.7,0.8,0.9,1.0$ the solution of the problem (4.4) has been obtained and tabulated in Table 1.

Table 1. values of the Objectives for choosen values of $\alpha$

\begin{tabular}{|c|c|c|c|c|c|c|c|c|}
\hline Values of $\alpha$ & $x_{1}$ & $x_{2}$ & $\left(Z_{1}\right)_{\alpha}$ & $\left(Z_{2}\right)_{\alpha}$ & $\left(W_{1}\right)_{\alpha}$ & $\left(W_{2}\right)_{\alpha}$ & $d$ & Sum of deviation \\
\hline \hline 0.1 & 46.223 & 41.528 & 715.591 & 301.171 & 75.292 & 179.664 & 0.50148 & 373.12 \\
\hline 0.2 & 42.871 & 34.471 & 602.290 & 261.111 & 98.025 & 161.577 & 0.50016 & 329.139 \\
\hline 0.3 & 39.830 & 28.623 & 506.788 & 228.018 & 94.215 & 145.495 & 0.50001 & 282.096 \\
\hline 0.4 & 37.097 & 23.858 & 423.995 & 200.277 & 88.985 & 130.799 & 0.50002 & 276.194 \\
\hline 0.5 & 34.615 & 20.454 & 336.608 & 179.371 & 85.752 & 120.343 & 0.500005 & 277.806 \\
\hline 0.6 & 32.354 & 16.871 & 305.985 & 159.783 & 79.593 & 108.572 & 0.500008 & 233.65 \\
\hline 0.7 & 30.242 & 14.342 & 259.805 & 143.915 & 74.702 & 99.207 & 0.50064 & 217.933 \\
\hline 0.8 & 28.379 & 12.237 & 220.629 & 130.647 & 69.984 & 91.021 & 0.5002 & 205.044 \\
\hline 0.9 & 26.624 & 10.518 & 187.452 & 119.118 & 65.540 & 83.750 & 0.50004 & 193.886 \\
\hline 1.0 & 26.249 & 9.091 & 161.59 & 114.087 & 62.613 & 79.771 & 0.5549 & 180.324 \\
\hline
\end{tabular}

From Table 1, it has been observed that with the increase of safety level $\alpha$, the sum of the deviations $(D)$ of the objective values from their respective ideal values diminishes which is shown in the last column of Table 1 and a minimum is obtained at $\alpha=1$ which denotes certainty (i.e. the situation where the parameters could be determined crisply). In real world situation, there is always some uncertainty associated with the parameters of the problem. The parameters are not all crisps. So the decision maker has to take some risk.

Here we take $\alpha=0.8$ which associates a risk factor $1-\alpha=0.2$, but the objective values are closer to their respective ideal values. From Table 1, using the objective values we can plot $(\alpha, Z)$ and $(\alpha, W)$ graphs (Figure 1$)$.

After putting $\alpha=0.8$, Example 1 is reduced to a standard De Novo problem. Its optimal solution has been given in Table 2. The solution obtained by Li and Lee [14] for this problem with $\alpha=0.8$ are also shown in this Table. A comparison shows that the two solutions are same.

Table 2. Comparison between the results obtained by the different methods

\begin{tabular}{|c|c|c|c|c|c|c|c|c|}
\hline \multirow{2}{*}{ Method } & \multirow{2}{*}{ DecisionVariable } & \multirow{2}{*}{ Value } & \multicolumn{4}{|c|}{ Value of Objectives } & \multirow{2}{*}{$d$} & \multirow{2}{*}{$\tilde{B}$} \\
\hline & & & $\left(Z_{1}\right)_{\alpha}$ & $\left(Z_{2}\right)_{\alpha}$ & $\left(\tilde{W}_{1}\right)_{\alpha}$ & $\left(\tilde{W}_{2}\right)_{\alpha}$ & & \\
\hline \multirow{2}{*}{$\mathrm{Li}$ and Lee } & $x_{1}$ & 28.38 & \multirow{2}{*}{220.7} & \multirow{2}{*}{130.7} & \multirow{2}{*}{70} & \multirow{2}{*}{91} & & \\
\hline & $x_{2}$ & 12.24 & & & & & & \\
\hline \multirow[t]{2}{*}{ Proposed method } & $x_{1}$ & $\begin{array}{l}28.379 \\
12237\end{array}$ & \multirow[t]{2}{*}{220.629} & \multirow[t]{2}{*}{130.647} & \multirow[t]{2}{*}{69.9848} & \multirow[t]{2}{*}{91.0216} & \multirow[t]{2}{*}{0.50002} & \multirow[t]{2}{*}{210} \\
\hline & $x_{2}$ & 12.237 & & & & & & \\
\hline
\end{tabular}

Next we consider another example having more objectives as well as decision variables. We solve it by our proposed method as well as that of $\mathrm{Li}$ and Lee, and compare the results for justification and worthiness of the approach proposed in this paper. 

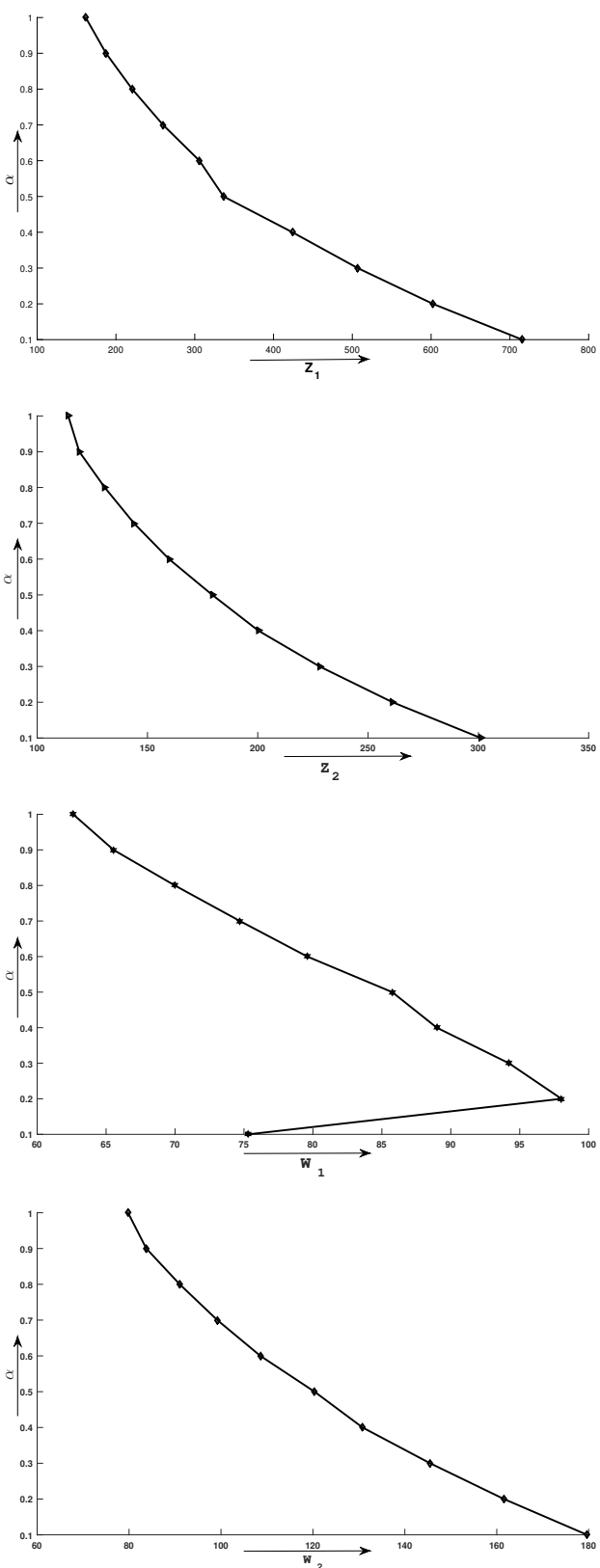

Figure 1. System designs for different values of $\alpha$

Example: 2

$$
\begin{array}{ll}
\operatorname{Max} & \tilde{Z}_{1}=\left[2^{0}, 3^{1}\right) x_{1}+5 x_{2}+\left[7^{0}, 8^{1}\right) x_{3}+x_{4} \\
\operatorname{Max} & \tilde{Z}_{2}=4 x_{1}+\left[1^{0}, 2^{1}\right) x_{2}+3 x_{3}+\left[11^{0}, 12^{1}\right) x_{4} \\
\operatorname{Max} & \tilde{Z}_{3}=\left[9^{0}, 10^{1}\right) x_{1}+3 x_{2}+\left[1^{0}, 3^{1}\right) x_{3}+2 x_{4} \\
\operatorname{Min} & \tilde{W}_{1}=1.5 x_{1}+\left(1^{1}, 2^{0}\right] x_{2}+0.3 x_{3}+\left(2^{1}, 3^{0}\right] x_{4} \\
\operatorname{Min} & \tilde{W}_{2}=0.5 x_{1}+x_{2}+0.73 x_{3}+2 x_{4}
\end{array}
$$

Subject to

$$
\begin{aligned}
& \left(1^{1}, 3^{0}\right] x_{1}+4.5 x_{2}+1.5 x_{3}+\left(7^{1}, 8^{0}\right] x_{2}=\left[150^{0}, 200^{1}\right) . \\
& \quad x_{1}, x_{2}, x_{3}, x_{4} \geq 0
\end{aligned}
$$


As in Example 1, here also we have converted this fuzzy programming into an equivalent crisp linear programming, as following:

$$
\begin{array}{ll}
\operatorname{Max} & \left(\tilde{Z}_{1}\right)_{\alpha}=(3-\alpha) x_{1}+5 x_{2}+(8-\alpha) x_{3}+x_{4} \\
\operatorname{Max} & \left(\tilde{Z}_{2}\right)_{\alpha}=4 x_{1}+(1-\alpha) x_{2}+3 x_{3}+(12-\alpha) x_{4} \\
\operatorname{Max} & \left(\tilde{Z}_{3}\right)_{\alpha}=(10-\alpha) x_{1}+3 x_{2}+(3-2 \alpha) x_{3}+2 x_{4} \\
\operatorname{Min} & \left(\tilde{w}_{1}\right)_{\alpha}=1.5 x_{1}+(1+\alpha) x_{2}+0.3 x_{3}+(2+\alpha) x_{4} \\
\operatorname{Min} & \left(\tilde{W}_{2}\right)_{\alpha}=0.5 x_{1}+x_{2}+0.73 x_{3}+2 x_{4}
\end{array}
$$

Subject to

$$
\begin{aligned}
& (1+2 \alpha) x_{1}+4.5 x_{2}+1.5 x_{3}+(7+\alpha) x_{4}=150-50 \alpha \\
& \alpha \in[0,1], \quad x_{1}, x_{2} \geq 0
\end{aligned}
$$

In this case also varying $\alpha \in[0,1]$, the solution and the corresponding objective values could be determined by Lingo and curves of the objective functions could be drawn. From this graph, the decision maker can choose the values of the objective as per his/her requirement and the corresponding safety level $\alpha$ and consequent risk factor $(1-\alpha)$ the decision maker is ready to concede. Here for $\alpha=0.8$ the program has been solved and a comparison of the optimal solution obtained by $\mathrm{Li}$ and Lee and our proposed approach is given in Table 3.

Table 3. Comparison between the results obtained by the different methods

\begin{tabular}{|c|c|c|}
\hline Objective Functions & Li and Lee Fuzzy Approach & Proposed Approach \\
\hline$\left(\tilde{Z}_{1}\right)_{\alpha}$ & 310.481 & 310.51616 \\
\hline$\left(\tilde{Z}_{2}\right)_{\alpha}$ & 194.608 & 194.608 \\
\hline$\left(\tilde{Z}_{3}\right)_{\alpha}$ & 245.901 & 245.932 \\
\hline$\left(\tilde{W}_{1}\right)_{\alpha}$ & 42.729 & 42.7306 \\
\hline$\left(\tilde{W}_{2}\right)_{\alpha}$ & 37.341 & 38.935 \\
\hline Closeness to the ideal & $d=0.57,0.88,0.603,0.5,0.5$ & $d=0.5000214$ \\
\hline \multirow{3}{*}{ Variables } & $x_{1}=21.14916$ & $x_{1}=21.15207$ \\
\cline { 2 - 3 } & $x_{2}=0$ & $x_{2}=0.000693$ \\
\cline { 2 - 3 } & $x_{3}=36.6598$ & $x_{3}=36.664$ \\
\cline { 2 - 3 } & $x_{4}=0.00288$ & $x_{4}=0.000797$ \\
\hline
\end{tabular}

From the Table 3, it is evident that both the methods generate same solution of the problem.

\section{Conclusion}

A one-step method for the solution of a general De Novo Programming Problem with the fuzzy parameters has been introduced in this paper. The solution procedure has been illustrated by numerical examples. The problems have been solved by our proposed approach as well as by the two-step fuzzy approach of Li and Lee. It is observed from the comparison table that both the methods yield the same solutions of the considered general De Novo Programming Problems with fuzzy parameters. This coincidence is due to fact that the proposed method in this paper elicits an efficient solution of the problem in one step (Theorem 4.1) and by the method of Li and Lee also the same could be obtained but in a two-step approach. But the proposed method is better because it yields the desired solutions in one-step only. It requires much fewer computations and processing time than the two-phase fuzzy method. In large problems, these benefits are 
of considerable importance. As a future scope of the study, the solution of general fuzzy De Novo Programming Problem,where the coefficients are other types of fuzzy numbers (e.g. triangular, trapezoidal) or other types of fuzzy sets (e.g. type-2 fuzzy set, hesitant fuzzy set) and using the different variants of goal programming could be investigated.

Acknowledgment. The authors would like to express their sincere thanks to the referee for his/her valuable suggestions regarding improvement of the paper in the present form.

\section{References}

[1] Z. Babic and I. Pavic Multicriterial production planning by de novo programming approach, Int. J. Prod. Econ. 43 (1), 59-66, 1996.

[2] C. Carlsson and P. Korhonen, A parametric approach to fuzzy linear programming, Fuzzy sets and systems, 20 (1), 17-30, 1986.

[3] S. Chackraborty and D. Bhattacharya, A new approach for solution of multi-stage and multi-objective decision-making problem using de novo programming, Eur. J. Sci. Res. 79 (3), 393-417, 2012.

[4] S. Chackraborty and D. Bhattacharya, Optimal system design under multi-objective decision making using de-novo concept: A new approach, Int. J. Comput. Appl. 63 (12), 20-27, 2013.

[5] A. Charnes and W.W. Cooper Management models and industrial applications of linear programming, Management Science, 4 (1), 38-91, 1957.

[6] J.K.C. Chen and G.-H. Tzeng, Perspective strategic alliances and resource allocation in supply chain systems through the de novo programming approach, Int. J. Sustain. Strat. Manag. 1 (3), 320-339, 2009.

[7] Y.-W. Chen and H.-E. Hsieh Fuzzy multi-stage de-novo programming problem, Appl. Math. Comput. 181 (2), 1139-1147, 2006.

[8] R.B. Flavell, A new goal programming formulation, Omega, 4 (6), 731-733, 1976.

[9] J.J. Huang, G.-H. Tzeng and C.-S. Ong, Choosing best alliance partners and allocating optimal alliance resources using the fuzzy multi-objective dummy programming model, J. Oper. Res. Soc. 57 (10), 1216-1223, 2006.

[10] J.P. Ignizio, Linear programming in single and multiple objective systems, PrenticeHall. Inc., Englewood Cliffs, New Jersey, 1982.

[11] Y. Ijiri. Management goals and accounting for control, North Holland Publication, 3, 1965.

[12] D.F. Jones and M. Tamiz, Goal programming in the period 1990 - 2000. In Multiple Criteria Optimization: State of the art annotated bibliographic surveys, 129-170, 2003.

[13] S.M. Lee, Goal programming for decision analysis, Auerbach Publishers, Philadelphia, 1972.

[14] R.J. Li and E.S. Lee, Fuzzy approaches to multicriteria de novo programs, J. Math. Anal. Appl. 153 (1), 97-111, 1990.

[15] R.J. Li and E.S. Lee, Multi-criteria de novo programming with fuzzy parameters, Comput. Math. Appl. 19 (5), 13-20, 1990.

[16] M.K. Luhandjula, Compensatory operators in fuzzy linear programming with multiple objectives, Fuzzy sets and systems, 8 (3), 245-252, 1982.

[17] D.Y. Miao, W.W. Huang, Y.P. Li and Z.F. Yang, Planning water resources systems under uncertainty using an interval-fuzzy de novo programming method, J. Environ. Inform. 24 (1), 11-23, 2014.

[18] C. Romero, Handbook of critical issues in goal programming, Elsevier, 2014.

[19] S. Saeedi, M. Mohammadi and S. Torabi A de novo programming approach for a robust closed-loop supply chain network design under uncertainty: An m/m/1 queueing model, Int. J. Ind. Eng. Comput. 6 (2), 211-228, 2015 
[20] Y. Shi Studies on optimum-path ratios in multicriteria de novo programming problems, Comput. Math. Appl. 29 (5), 43-50, 1995.

[21] Y. Shi Optimal system design with multiple decision makers and possible debt: a multicriteria de novo programming approach, Oper. Res. 44 (5), 723-729, 1999.

[22] N. Umarusman, Min-max goal programming approach for solving multi-objective de novo programming problems, Int. J. Oper. Res. 10, 92-99, 2013.

[23] J.L. Verdegay, A dual approach to solve the fuzzy linear programming problem, Fuzzy sets and systems, 14 (2), 131-141, 1984.

[24] L.A. Zadeh, Fuzzy sets as a basis for a theory of possibility, Fuzzy sets and systems, 100, 9-34, 1999.

[25] M. Zeleny, Multi-objective design of high-productivity systems, Joint Automatic Control Conference-Paper APPL9-4, ASME, Newyork, 13, 297-300, 1976.

[26] M. Zeleny (Ed), Mathematical programming with multiple objectives(special issue), Comput. Oper. Res. 7, 101-107, 1980.

[27] M. Zeleny, A case study in multi-objective design: De novo programming, Multiple Criteria Analysis: Operational Methods, (Edited by P. Nijkamp and J. Spronk), Gower publishing Co., Hampshire, 37-52, 1981.

[28] M. Zeleny, On the squandering of resources and profits via linear programming, Interfaces, 11 (5), 101-107, 1981.

[29] M. Zeleny, Optimal system design with multiple criteria: De novo programming approach, Eng. Cost. Prod. Econ. 10 (2), 89-94, 1986.

[30] M. Zeleny, Optimizing given systems vs. designing optimal systems: The de novo programming approach, Int. J. Gen. Syst. 17 (4), 295-307, 1990.

[31] Y.M. Zhang, G.H. Huang and X.D. Zhang. Inexact de novo programming for water resources systems planning, European J. Oper. Res. 199 (2), 531-541, 2009.

[32] H.J. Zimmermann, Fuzzy programming and linear programming with several objective functions, Fuzzy sets and systems, 1 (1), 45-55, 1978. 